\title{
Entamoeba species associated with chronic diarrhoea in Pakistan
}

\author{
J. YAKOOB ${ }^{1 *}$, Z. ABBAS ${ }^{1}$, M. A. BEG ${ }^{2}$, S. NAZ ${ }^{1}$, R. KHAN ${ }^{1}$ AND W. JAFRI ${ }^{1}$ \\ ${ }^{1}$ Department of Medicine and \\ ${ }^{2}$ Microbiology and Pathology, The Aga Khan University, Karachi, Pakistan
}

(Accepted 28 January 2011; first published online 14 March 2011)

\section{SUMMARY}

We determined the prevalence of Entamoeba (E.) histolytica, E. dispar and E. moshkovskii in patients with chronic diarrhoea associated with abdominal pain or discomfort mimicking irritable bowel syndrome. Stool samples were collected from 161 patients with chronic diarrhoea and from 157 healthy controls. Stool microscopy with modified trichrome stain, culture and polymerase chain reaction (PCR) for Entamoeba spp. differentiation was performed. Microscopy demonstrated Entamoeba cysts in $44 \%$ (57/129) of patients with diarrhoea compared to $29 \%$ $(44 / 151)$ of controls $(P=0 \cdot 009)$. In patients with diarrhoea, PCR for $E$. histolytica was positive in $9 \%(11 / 129)(P=0 \cdot 008)$, E. dispar in $19 \%(24 / 129)(P=0 \cdot 117)$ and E. moshkovskii in $19 \%$ $(24 / 129)(P<0 \cdot 001)$. E. histolytica and E. moshkovskii were significantly associated with diarrhoea while $E$. dispar was found equally in both groups.

Key words: Diarrhoea, Entamoeba histolytica, Entamoeba moshkovskii, Entamoeba dispar, PCR.

\section{INTRODUCTION}

Intestinal amoebiasis is caused by the protozoan Entamoeba (E.) histolytica, a non-flagellated amoeboid protozoan parasite. E. histolytica is an invasive pathogen commonly acquired in the developing world. However, most humans infected with E. histolytica are asymptomatic [1]. When clinical symptoms develop, they are usually limited to the gastrointestinal tract. The symptoms of abdominal pain, tenderness and diarrhoea may mimic manifestation of irritable bowel syndrome (IBS) or inflammatory bowel disease. E. histolytica has the ability to lyse host cells and cause tissue destruction while it also

\footnotetext{
* Author for correspondence: Dr J. Yakoob, MBBS, PhD, FACP, Department of Medicine, The Aga Khan University, PO Box 3500, Stadium Road, Karachi-74800, Pakistan.

(Email: javed.yakoob@aku.edu)
}

induces both cellular and humoral immune responses in extraintestinal disease [2].

The genus Entamoeba, including species E. histolytica, E. dispar, E. moshkovskii, E. poleki, E. coli and $E$. hartmanni may colonize the human intestinal lumen. E. histolytica is known to cause intestinal and extraintestinal disease while other species are regarded as commensal organisms that cause no intestinal disease. Faecal carriage of E. dispar is more common than E. histolytica. It is known that even in areas where invasive amoebiasis is common E. dispar is the more prevalent species [3]. Mixed infection with E. histolytica, E. dispar and/or E. moshkovskii have been reported [4]. The demonstration of cysts or trophozoites in the stool suggests an intestinal amoebic infection, but microscopy cannot differentiate between E. histolytica and E. dispar or E. moshkovskii.

Amoebiasis may form part of the differential diagnosis of IBS, especially in patients with acute 
exacerbations of IBS symptoms. Early studies implicated amoebic dysentery in the development of IBS in British soldiers returning from Egypt at the end of the Second World War [5, 6]. However, in later years, several studies refuted this assertion and exposure to E. histolytica was thought not to predispose patients to IBS and all patients spontaneously eradicated the organism [7-9]. In the current study, the prevalence of E. histolytica, E. dispar and E. moshkovskii was determined in patients who presented with IBS, i.e. chronic diarrhoea associated with abdominal pain and or discomfort.

\section{MATERIALS AND METHODS}

\section{Patients}

A total of 318 stool samples were examined between June 2008 and December 2009. They were obtained from 161 patients with chronic intermittent diarrhoea for the last 3 months associated with abdominal discomfort or pain mimicking IBS and $\geqslant 3$ loose stool per day, and 157 healthy controls with normal bowel habits who volunteered stool samples. Patients with IBS symptoms attended the gastroenterology clinic at the Aga Khan University, Karachi. Their mean age was $41 \pm 15$ (range 16-83) years with a male:female ratio of 112:49. These patients underwent history, physical examination, complete blood count, serum creatinine, electrolytes, stool microscopy, culture and polymerase chain reaction (PCR) for E. histolytica, E. dispar and E. moshkovskii. Presence of chronic diseases, e.g. celiac disease, thyroid dysfunction and chronic pancreatitis were ruled out by tissue transglutaminase antibody IgA and IgG, thyroid-stimulating hormone and serum amylase level. In the control group, there were 157 healthy individuals without previous history of diarrhoea. They were comprised of medical and paramedical staff members who volunteered for a history, physical examination and a stool sample. Their age and sex closely matched the patient group with diarrhoea. Controls were all local residents of Karachi and randomly selected. They were excluded if they had a history of diarrhoea, foreign travel or were exposed to risk factors for infection during the last 6 weeks; they were known not to suffer from any comorbid illnesses that could contribute to chronic infection. The study was approved by the institutional ethics review committee of the Aga Khan University. All stool specimens were processed by microscopy and culture for Entamoeba and the presence of other parasites such as Blastocystis hominis, Giardia lamblia, E. coli, E. hartmanni, etc. was noted. DNA used for PCR was extracted from both unpreserved stool specimen and Entamoeba culture to ensure that the organism which grew was the same as that seen by the microscope or detected by PCR. The Entamoeba genus-specific primers were first used with extracted DNA to detect Entamoeba spp. which was followed by PCR with species-specific primers to detect E. histolytica, E. dispar and E. moshkovskii.

A microbiological investigation was also performed to detect Salmonella spp., Shigella sonnei, Campylobacter jejuni, Clostridium difficile and Vibrio cholerae. However, a viral screen was not performed on stool specimens.

\section{Microscopy of stool smear}

Stool sample microscopy consisted of examining $\sim 2 \mathrm{mg}$ emulsified faeces with one drop of physiological saline and Lugol's iodine covered with a coverslip on two separate glass slides. Later an ethanol-fixed faecal smear was stained with modified trichrome stain. These preparations were examined under both low $(\times 10)$ and high $(\times 40-100)$ power. The diameter of the cysts was measured and the nuclei were counted. A 4-nucleated cyst having a diameter ranging from 10 to $16 \mu \mathrm{m}$ was identified as an Entamoeba cyst.

\section{Culture of stool specimen}

Cultures were performed for Entamoeba spp. in Robinson's medium shortly after collection as previously described [10]. The cultures were incubated at $37^{\circ} \mathrm{C}$ and examined after $2-3$ days. The culture was deemed negative if no growth was observed after 5 days. The sediment was examined as described above.

\section{Extraction of genomic DNA}

Stool DNA was extracted by using Stool DNA Extraction kit (Qiagen, USA) according to the manufacturer's protocol. Extracted DNA was stored at $-20{ }^{\circ} \mathrm{C}$ until PCR was performed for Entamoeba spp.

\section{PCR for Entamoeba spp.}

The primers used were as previously described (Table 1) [11]. These primers detected nucleotide 
Table 1. Primer sequences used for PCR

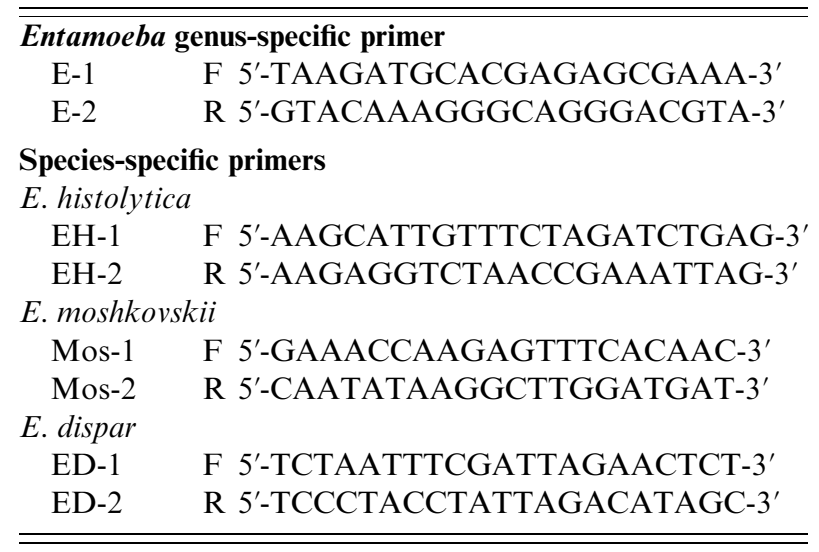

Source: Khairnar \& Parija [18].

sequences of 16S-like ribosomal RNA gene (16S rRNA) of E. histolytica, E. dispar and E. moshkovskii. For genus-specific and species-specific PCR, the reaction volume of $25 \mu \mathrm{l}$ comprised $2.5 \mu \mathrm{l}$ of $10 \times \mathrm{PCR}$ buffer (Promega, USA), $2 \cdot 0 \mu \mathrm{l}$ of $25 \mathrm{~mm} \mathrm{MgCl}_{2}$ (Promega), $0.75 \mu$ deoxyribonucleotide triphosphate mix (10 mm each dNTP, Promega), $0 \cdot 5 \mu \mathrm{l}$ (5 IU/ $\mu \mathrm{l})$ Taq polymerase (Promega), $0.25 \mu \mathrm{M}$ primers (IDT) and $2 \cdot 0 \mu \mathrm{l}$ template DNA. Amplification was performed in a PerkinElmer 9700 thermal cycler with both positive and negative controls. Positive controls consisted of DNA that has been positive twice for the same Entamoeba species while distilled water was used as the negative control. The PCR conditions consisted of one cycle denaturing at $94{ }^{\circ} \mathrm{C}$ for $5 \mathrm{~min}, 35$ cycles including annealing at $55^{\circ} \mathrm{C}$ for $1 \mathrm{~min}$, extension at $72{ }^{\circ} \mathrm{C}$ for $1 \mathrm{~min}$, denaturing at $94{ }^{\circ} \mathrm{C}$ for $1 \mathrm{~min}$, and an additional cycle with a 5 -min chain elongation at $72{ }^{\circ} \mathrm{C}$ (PCR System 9700, PerkinElmer, USA). The PCR products and molecular markers were electrophoresed in 2\% agarose gel with Tris-acetate-EDTA electropheresis buffer. The size markers were 100-bp ladders (Promega). The PCR amplification for each primer pair was repeated at least three times. Bands were visualized by the imaging system (Gel Doc 2000, Gel Documentation System, Bio-Rad, UK) after being stained with ethidium bromide.

\section{Statistical method}

Results were expressed as mean \pm standard deviation for continuous variables (e.g. age) and number (percentage) for categorical data (e.g. gender, stool culture, diarrhoea, etc.). Univariate analysis was performed by using the independent-sample $t$ test,
Pearson's $\chi^{2}$ test and Fisher's exact test where appropriate. The kappa $(\kappa)$ test was used to compare methods. A $P$ value $<0.05$ was considered statistically significant. All $P$ values were two-sided. Statistical interpretation of data was performed by using the computerized software program SPSS version 16.0 (SPSS Inc. USA).

\section{RESULTS}

Stool microscopy with modified trichrome stain for Entamoeba cyst was positive in 36\% (115/318) of samples. Entamoeba culture was positive in $38 \%$ (121/318). Entamoeba genus PCR was positive in 39\% (125/318) with E. dispar positive in $19 \%(59 / 318)$, E. moshkovskii in $13 \%(42 / 318)$ and E. histolytica in $7 \%(21 / 318)$, while it was non-typable in $3 \%(8 / 318)$ of samples. Co-infection with $B$. hominis was seen in $12 \%(38 / 318)$ of samples by stool culture. These comprised of $20 \%(32 / 161)$ of patients and $4 \%$ $(6 / 157)$ in the control group. These patients with B. hominis co-infection were excluded and the final analysis was performed in 280 patients, $129(46 \%)$ with diarrhoea and 151 (54\%) controls. Endolimax nana cysts were noted in six (2\%) samples which were equally present in both groups. Bacterial cultures were negative in these patients for Salmonella spp., Sh. sonnei, C. jejuni, Cl. difficile and V. cholerae.

\section{Association of symptoms with Entamoeba spp.}

Patients with diarrhoea had E. histolytica infection $(9 \%, 11 / 129)$ compared to $1 \%(2 / 151)$ of controls $(P=0.004)$ while $19 \%(24 / 129)$ of patients had E. moshkovskii infection compared to $4 \%(6 / 151)$ of controls $(P<0 \cdot 001)$ (Table 2). E. dispar was present in $19 \%(24 / 129)$ of patients with diarrhoea compared to $27 \%(40 / 151)$ of controls $(P=0 \cdot 679)$.

\section{Diagnostic yield of various tests used for the diagnosis of Entamoeba spp.}

Stool microscopy with staining demonstrated Entamoeba cysts in $44 \%(57 / 129)$ of patients with diarrhoea compared to $(44 / 151)$ of controls $(P=0 \cdot 009)$. Entamoeba culture was positive in $36 \%(47 / 129)$ of patients compared to $27 \%(40 / 151)$ of controls $(P=0 \cdot 073)$. One patient had mixed infection with E. moshkovskii and E. dispar. PCR for Entamoeba genus was positive in $46 \%(59 / 129)$ of patients compared to $33 \%(49 / 151)$ of controls $(P=0 \cdot 023)$. 
Table 2. Details of patients included in the study

\begin{tabular}{|c|c|c|c|}
\hline & $\begin{array}{l}\text { Diarrhoea cases } \\
n=129(46)\end{array}$ & $\begin{array}{l}\text { Controls } \\
n=151(54)\end{array}$ & $P$ value \\
\hline \multicolumn{4}{|l|}{ Age (yr) } \\
\hline Mean \pm s.D. & $41 \pm 15$ & $42 \pm 14$ & \\
\hline Range & $16-83$ & $15-75$ & \\
\hline \multicolumn{4}{|l|}{ Gender } \\
\hline Male & $87(67)$ & $99(66)$ & $0 \cdot 704$ \\
\hline Female & $42(33)$ & $52(34)$ & \\
\hline \multicolumn{4}{|c|}{ Stool microscopy } \\
\hline Positive & $57(44)$ & $44(29)$ & $0 \cdot 009$ \\
\hline Negative & $72(56)$ & $107(71)$ & \\
\hline \multicolumn{4}{|c|}{ Culture for Entamoeba } \\
\hline Positive & $47(36)$ & $40(27)$ & 0.073 \\
\hline Negative & $82(64)$ & $111(73)$ & \\
\hline \multicolumn{4}{|c|}{ Entamoeba genus PCR } \\
\hline Positive & $59(46)$ & $49(33)$ & $0 \cdot 023$ \\
\hline Negative & $70(54)$ & $102(67)$ & \\
\hline \multicolumn{4}{|c|}{ Genus-specific PCR } \\
\hline \multicolumn{4}{|c|}{ E. histolytica } \\
\hline Positive & $11(9)$ & $2(1)$ & $0 \cdot 004$ \\
\hline Negative & $118(91)$ & $149(99)$ & \\
\hline \multicolumn{4}{|l|}{ E. dispar } \\
\hline Positive & 24 (19) & $40(27)$ & $0 \cdot 117$ \\
\hline Negative & $105(81)$ & $111(73)$ & \\
\hline \multicolumn{4}{|l|}{ E. moshkovskii } \\
\hline Positive & 24 (19) & $6(4)$ & $<0.001$ \\
\hline Negative & $105(81)$ & $145(96)$ & \\
\hline \multicolumn{4}{|c|}{$\begin{array}{l}\text { Entamoeba genus PCR was positive but specific PCR for the three species } \\
\text { was negative }\end{array}$} \\
\hline Positive & $4(3)$ & $5(3)$ & $0 \cdot 921$ \\
\hline Negative & $125(97)$ & $146(97)$ & \\
\hline
\end{tabular}

Univariate analysis was performed by using the independent-sample $t$ test, Pearson's $\chi^{2}$ test and Fisher's exact test were also used whenever appropriate. A $P$ value $<0.05$ was considered statistically significant.

Values are number and percentage: $n(\%)$.

E. histolytica was positive in $9 \%(11 / 129)$ of patients compared to $1 \%(2 / 151)$ of controls $(P=0.008)$ (Table 2). E. moshkovskii was positive in $19 \%$ $(24 / 129)$ of patients compared to $4 \%(6 / 151)$ of controls $(P<0 \cdot 001)$ (Table 2$)$.

\section{Correlation between stool microscopy and PCR for Entamoeba spp.}

Entamoeba cysts were observed by stool microscopy in $44 \%(57 / 129)$ of patients with diarrhoea while PCR for E. histolytica was positive in 9\% (11/129) $(\kappa=0 \cdot 14, \quad P<0 \cdot 001), \quad E$. dispar in $19 \%(24 / 129)$ $(\kappa=0.21, P=0.004)$ and E. moshkovskii in $19 \%$ (24/129) $(\kappa=0 \cdot 21, P=0 \cdot 004)$, respectively, compared to when Entamoeba cysts were negative $(56 \%, 72 / 129)$.

\section{DISCUSSION}

Clinical amoebiasis may mimic functional bowel disease when it has a subacute onset with symptoms of mild diarrhoea and abdominal pain [12]. E. dispar and E. moshkovskii infections and $90 \%$ of E. histolytica infections were reported as being asymptomatic [12]. This study showed that in patients with diarrhoea and abdominal pain, Entamoeba cysts were commonly seen on stool microscopy. Diarrhoea was associated with both E. histolytica and E. moshkovskii infection. These patients were treated with metronidazole and diloxanide combination following which they had symptomatic improvement with a clear repeat stool examination. In our study we did not use a concentration step such as formol-ether centrifugation which 
is known to increase the sensitivity of microscopy as the diarrhoeal stool samples were usually liquid and did not concentrate well. The direct stool smear technique which is quick and inexpensive was preferred for the observation of motile protozoan trophozoites. It is possible that by not using a concentration technique, we may have missed some parasites if their concentration was low or if much debris was present. We did not anticipate any difference in the sensitivity of stool testing based on its consistency between cases and controls, as most of our cases described their stool consistency as loose and not watery. Further, clinical details of the patient were not known at the time of stool microscopic examination. The yield of stool culture and Entamoeba genus PCR was better than microscopy. E. dispar species was present equally in both groups. Only two patients with diarrhoea had infection with two different types of Entamoeba spp. In these cases, E. moshkovskii was associated with E. histolytica or E. dispar. E. histolytica was significantly associated with diarrhoea and was found in only one in healthy control who did not have a history of diarrhoea (Table 2). In a few cases, we were unable to type the Entamoeba species which might represent $E$. coli and E. hartmanni that are common commensals in the intestinal tract of the humans.

In this study, E. moshkovskii was demonstrated as the second most common Entamoeba spp. E. dispar is described as a non-pathogen although it has been shown to be capable of producing variable focal intestinal lesions in animals and destroyed epithelial cell monolayers in vitro [13-15]. E. moshkovskii cysts are morphologically indistinguishable from those of E. histolytica and E. dispar. In a previous study, prevalence of E. moshkovskii infection of $21 \%$ was reported in children aged 2-5 years in Bangladesh [4]. E. dispar-infected children were twice as likely to be co-infected with E. moshkovskii (35\%) compared to those with E. histolytica (18\%) infection [4]. A study in India has also linked E. moshkovskii infection with dysentery [16]. Similarly, in a study from Australia that did not use a control group, the prevalence of E. moshkovskii infection was $61 \cdot 8 \%$ in homosexual men and all these patients were symptomatic [17].

This is the first report to study the molecular typing of Entamoeba spp. in Pakistan and highlights the incidence of co-infection in our patients. It is important to recognize that routine stool microscopic examination techniques do not impart complete information to allow differentiatiation between the Entamoeba spp. Co-infection with Entamoeba spp. might be responsible for exacerbation of symptoms in some of the known IBS cases. The reporting of Entamoeba cysts by stool microscopy might not be suggestive of a benign course. There might be co-infection with more than one type of Entamoeba spp. that could not be identified on routine stool microscopic examination. It is possible that $E$. dispar in association with E. moshkovskii may contribute to the development of diarrhoea.

\section{ACKNOWLEDGEMENTS}

We are grateful to the staff of the Juma Research Laboratory for their help during the completion of this work.

\section{DECLARATION OF INTEREST}

None.

\section{REFERENCES}

1. Benetton ML, et al. Risk factors for infection by E. histolytica/E. dispar complex: an epidemiological study conducted in outpatient clinics in the city of Manaus, Amazon Region, Brazil. Transaction of Royal Society of Tropical Medicine and Hygiene 2005; 99: 532-540.

2. Ackers JP, Mirelman D. Progress in research on Entamoeba histolytica pathogenesis. Current Opinion Microbiology 2006; 9: 367-373.

3. Petri WA, Singh U. Enteric amebiasis. In: Guerrant R, Walker DH, Weller PF, eds. Tropical Infectious Diseases: Principles, Pathogens, and Practice. Philadelphia: Elsevier, 2006, 967 pp.

4. Ali IK, et al. Entamoeba moshkovskii infections in children, Bangladesh. Emerging Infectious Diseases 2003; 9: 580-584.

5. McMillan A, et al. Amebiasis in homosexual men. Gut 1984; 25 : 356-360.

6. Stewart GT. Post-dysenteric colitis. British Journal of Medicine 1950; 1: 405-409.

7. Chaudhary NA, Truelove SC. The irritable colon syndrome; a study of the clinical features, predisposing causes and prognosis in 130 cases. Quarterly Journal of Medicine $1962 ; 31$ : 307-322.

8. Anand AC, et al. Does nondysenteric intestinal amoebiasis exist? Lancet 1997; 349: 89-92.

9. Sinha $\mathbf{P}$, et al. Does Entamoeba histolytica cause irritable bowel syndrome? Indian Journal of Gastroenterology 1997; 16: 130-133.

10. Robinson GL. The laboratory diagnosis of human parasitic amoeba. Transactions of the Royal Society of Tropical Medicine and Hygiene 1968; 62: 285-294. 
11. Khairnar P, Khairnar SC. Detection of excretory Entamoeba histolytica DNA in the urine, and detection of E. histolytica DNA and lectin antigen in the liver abscess pus for the diagnosis of amoebic liver abscess. BMC Microbiology 2007; $7: 41$.

12. World Health Organization. Amebiasis. WHO Weekly Epidemiology Record 1997; 72: 97-100.

13. Chadee K, Smith JM, Meerovitch E. Entamoeba histolytica: electrophoretic isoenzyme patterns of strains and their virulence in the cecum of gerbils (Meriones unguiculatus). American Journal of Tropical Medicine and Hygiene 1985; 34: 870-878.

14. Espinosa-Cantellano M, Castanon-Gutierrez G, Martínez-Palomo A. In vivo pathogenesis of Entamoeba dispar. Archives of Medical Research 1997; 28: S204S206.
15. Espinosa-Cantellano M, et al. Entamoeba dispar: ultrastructure, surface properties, and cytopathic effect. Journal of Eukaryote Microbiology 1998; 45: 265-272.

16. Parija SC, Khairnar K. Entamoeba moshkovskii and Entamoeba dispar-associated Infections in Pondicherry, India. Journal of Health Population Nutrition 2005; 23: 292-295.

17. Fotedar R, et al. PCR detection of Entamoeba histolytica, Entamoeba dispar, and Entamoeba moshkovskii in stool samples from Sydney, Australia. Journal of Clinical Microbiology 2007; 45: 1035-1037.

18. Khairnar K, Parija SC. A novel nested multiplex polymerase chain reaction (PCR) assay for differential detection of Entamoeba histolytica, E. moshkovskii and E. dispar DNA in stool samples. BMC Microbiology 2007; 7: 47. 